\title{
Accidental intravenous bolus infusion of potassium chloride in a young man with hypokalemic periodic paralysis
}

\author{
Seyed Hesam Rahmani ${ }^{1 *}$, Gholamreza Faridaalaee² \\ 'Emergency Medicine Department, Imam Khomeini Hospital, Urmia University of Medical Sciences, Urmia, Iran \\ ${ }^{2}$ Emergency Medicine Department, Maragheh University of Medical Sciences, Maragheh, Iran
}

\author{
Received: 8 September 2017 \\ Accept: 12 November 2017 \\ Published online: 21 November 2017 \\ *Corresponding author: Seyed \\ Hesam Rahmani, Emergency Medicine \\ Department, Imam Khomeini Hospital, \\ Urmia university of Medical Sciences, \\ Urmia, Iran. Tel: +989121167809; Fax: \\ 041133858859; Email: rahmani_h@ \\ umsu.ac.ir \\ Competing interests: None. \\ Funding information: None. \\ Citation: Rahmani SH, Faridaalaee G. \\ Accidental intravenous bolus infusion \\ of potassium chloride in a young man \\ with hypokalemic periodic paralysis. \\ Journal of Emergency Practice \\ and Trauma 2019; 5(1): 29-31. doi: \\ $10.15171 /$ jept.2017.34
}

\begin{abstract}
Hypokalemic periodic paralysis is anautosomal dominantdisease characterized by muscle weakness or paralysis with a matching fall in blood potassium levels. Paralysis attacks often occur in adolescence and are induced by strenuous exercise followed by rest, high carbohydrateor high sodiummeal content, sudden changes in temperature, and even excitement, noise, flashing lights and cold temperatures. Despite global daily use of intravenous and oral potassium in the treatment of patients, data about intravenous bolus potassium is rare. Here we report a 29-year-old man complaining of weakness in the upper and lower limbs. By initial diagnosis of periodic hypokalemic paralysis, potassium chloride was ordered by oral route. After a few minutes, we heard patient screaming in pain. He complained of severe acute pain in peripheral intravenous line, palpitation and dyspnea. We noticed that potassium chloride vial was infused directly via cubital vein in few minutes erroneously. Physical exam revealed sinus tachycardia and hypertension. We began hyperkalemia treatment immediately. With appropriate treatment,the patient was discharged with good condition after 12 hours of admission.

Keywords: Hypokalemic paralysis, Hyperkalemia, Potassium chloride
\end{abstract}

\section{Introduction}

Hypokalemic periodic paralysis is an autosomal dominant disease characterized by muscle weakness or paralysis with a matching fall in serum potassium levels. Attacks often occur in adolescence and are induced by strenuous exercise, high carbohydrate or high sodium meal content, sudden changes in temperature and cold temperatures. Weakness may be mild and limited to certain muscle groups, or more severe full body paralysis. Attacks may last for a few hours or several days. Some people only develop symptoms of periodic paralysis due to hyperthyroidism (1). People with hypokalemic periodic paralysis maybe misdiagnosed as having a conversion disorder since the weakness is muscle-based and does not correspond to any nerve or spinal root distributions (2). Treatment of hypokalemic periodic paralysis focuses on relieving acute symptoms and preventing further attacks by avoiding carbohydrate-rich meals and strenuous exercise. Paralysis attacks can be managed by drinking one of various potassium salts dissolved in water. Rapidly absorbed boluses of liquid potassium are generally needed to stop an attack, but some patients also find maintenance results with time-released tablets. Intravenous potassium is seldom justified unless the patient is unable to swallow (3). Potassium chloride is extensively used as a potassium supplement, mostly in the form of salt substitute. Therapeutically, both oral and intravenous forms of potassium are used. Treatment of hypokalemia in stable patients who are able to tolerate oral intake can be done orally by food rich in potassium, salt substitutes and potassium supplements. IV replacement is appropriate for severe hypokalemia and infusion rate should be no greater than $40 \mathrm{mEq} / \mathrm{h}$. Cardiac manifestations are the most serious side effect of hyperkalemia and rapid KCL infusion, cardiac dysrhythmia such as VF, complete heart block and asystol may also occur (4). Here, we present the case of intravenous bolus injection of potassium chloride into cubital vein in a young man with hypokalemic periodic paralysis.

\section{Case report}

A 29-year-old healthy man was admitted to the emergency department (bodyweight, $78 \mathrm{~kg}$ ) complaining of 
the weakness in the upper and lower limbs. On arrival, the patient was hemodynamically stable. Oral temperature was $37.5^{\circ} \mathrm{C}\left(99.5^{\circ} \mathrm{F}\right)$, pulse rate $78 \mathrm{bpm}$ with a sinus rhythm, blood pressure $125 / 75 \mathrm{~mm} \mathrm{Hg}$, and his respiratory rate was 14 breaths per minute. The abdomen and bowel sounds were normal. The initial oxygen saturation was $94 \%$ (in room air). He was alert and awake with no apparent distress. In clinical examination, forces of upper limbs were $3 / 5$ but lower limbs were $4 / 5$. Weakness of upper limbs was more significant. By initial diagnosis of periodic hypokalemic paralysis, potassium chloride (50 cc of $15 \%$ [7.5 g]) was ordered by oral route. After a few minutes, we heard patient screaming in pain. We quickly attended the patient's bed and it was very surprising to see him severely agitated with severe acute pain in peripheral IV line, palpitation and dyspnea. Immediately, we found that all of potassium chloride vial was infused directly via right cubital vein in few minutes erroneously. After that, the patient was transferred to CPR part immediately. He went under cardiac monitoring and received oxygen by nasal canola. He had sinus tachycardia $(\mathrm{HR}=140)$, HTN (160/90) and tachypnea (RR: 28). Calcium gluconate solution (10 cc of 10\%) was intravenously injected in 2 minutes and one litre of normal saline was immediately infused. Only 15-20 minutes after injection of calcium gluconate and serum, dyspnea and palpitation became better, but 20 minutes latter symptoms of the patient flared up. Due to this situation another calcium gluconate solution was intravenously reinjected and one litre of normal saline was ordered again for one hour. The color Doppler sonography of the right upper extremity showed no thrombosis in superficial veins. Muscle weakness was healed in the first few minutes. A 12-lead electrocardiogram revealed sinus tachycardia at a rate of $140 \mathrm{bpm}$ (Figure 1). Lab data in admission time: a complete blood count in patient was normal except for a mildly elevated white blood cell count of $11.2 \times 103 / \mu \mathrm{L}(11.2 \times 109 / \mathrm{L})$, with $50 \%$ neutrophils, $48 \%$ lymphocytes, $1 \%$ monocytes, and $1 \%$ eosinophils, hemoglobin $15.3 \mathrm{~g} / \mathrm{dL}$, hematocrit $44.5 \%$. His serum blood urea nitrogen, creatinine, creatinine kinase, and liver function tests were normal (sodium $141 \mathrm{mEq} / \mathrm{L}$, potassium 3.2 $\mathrm{mEq} / \mathrm{L}$, calcium [total] $9 \mathrm{mg} / \mathrm{dL}$, magnesium $1.7 \mathrm{mg} / \mathrm{dL}$ ). Serum glucose was $130 \mathrm{mg} / \mathrm{dL}$. In the blood sample taken after the first injection of calcium gluconate, potassium was 4 and 3 hours before discharge, potassium level was

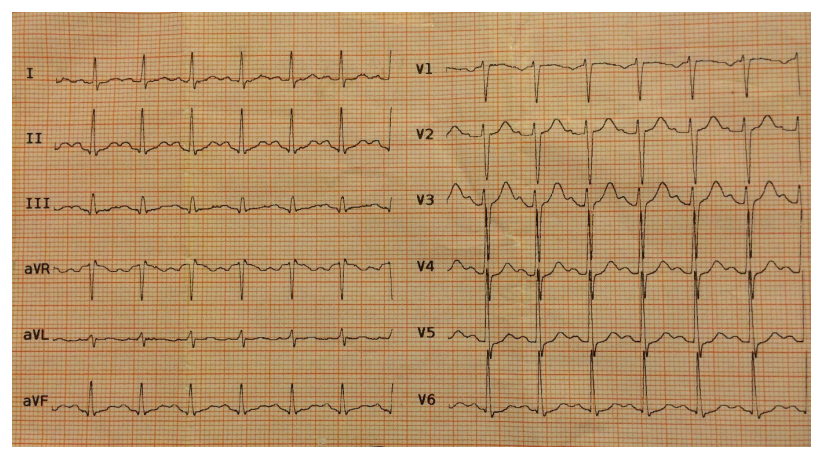

Figure 1. Twelve-lead electrocardiogram revealed sinus tachycardia.
3.8. Patient was discharged 12 hours after admission by diagnosis of periodic hypokalemic paralysis with recommendation of low carbohydrate and high potassium diet. He was advised to apply warm compress and ibuprofen $400 \mathrm{mg}$ orally every 6 hours was prescribed as well.

\section{Discussion}

Intentional intravenous potassium intoxication or intoxication due to medical treatment is a rare and very dangerous condition. The risks of intravenous potassium chloride injections are well known. Rapid or high dose injection can cause cardiac arrest within minutes. The effect of hyperkalemia on the heart is complex - virtually any arrhythmia may be observed (5). Hyperkalemia cause various electrocardiography (ECG) changes, depending on potassium serum concentration. So, ECG is essential to detect and follow these abnormalities. There is a close correlation between plasmatic potassium concentration and ECG changes with great variability (6). Rapid diagnosis and treatment of intravenous potassium poisoning by administration of calcium gluconate and other medications as beta-2-adrenergic agonists and insulin-glucose improved outcome (6). The ion exchange resins and diuretics have no role in this type of care. Their delayed effect dedicate them to the treatment of chronic moderate hyperkalemia. Hemodialysis performed in emergency is the standard treatment of hyperkalemia, particularly in cases of severe hyperkalemia which quickly set up or with high risk of reconstitution (hemolysis, tissue catabolism) (6). Most toxic hyperkalemia described in texts and case reports are due to sustained-release potassium supplements, side effects, or overdose of medical treatments (7-9).The true incidence of potassium-related fatalities and incidents is unknown. Fatal intravenous injection of potassium produces no specific anatomic changes and if any findings, it is subtle (10). Nilsson et al reported a case of a 30-yearold woman who was admitted to the emergency department due to the ingestion of 300 slow-release tablets of potassium-chloride. Her serum potassium was $9.5 \mathrm{mmol} / \mathrm{l}$ and cardiovascular function was poor. Despite intensive medical treatment and gastric lavage, serum potassium remained very high $(9 \mathrm{mmol} / \mathrm{l})$. Although hemodialysis was initiated but despite ongoing dialysis, potassium increased to $10.3 \mathrm{mmol} / \mathrm{l}$. A parallel dialysis was started and after 4 hours of parallel dialysis, serum potassium decreased to $6.4 \mathrm{mmol} / \mathrm{l}$. An x-ray revealed large amounts of pills remaining in the stomach and the surgeon removed about 200 pills with laparotomy. The patient recovered slowly thereafter, but later developed a gastric stricture and PTE (11). Battefort et al in 2012 reported a case of a 20 -year-old nurse student with IV injection of four vials of potassium chloride $(20 \mathrm{~mL}, 10 \%)$ and subsequent cardiac arrest with asystol rhythm. Initial resuscitation with epinephrine and sodium bicarbonate resulted in the recovery and effective cardiac activity without any squeal. They concluded that the advanced resuscitation with the use of a specific treatment helped to resuscitate the patient (12). Bertol et al in 2012 reported a case of a 41-year-old man who died due 
to intravenous injection of potassium chloride (13). In our case with underlying hypokalemia, sins tachycardia and no renal dysfunction it seems that hyperkalemia will be transient and we prefer to use just calcium gluconate to protect cardiac cell and hydration. We avoid using other treatment options such as insulin-glucose, beta-2-adrenergic agonists and bicarbonate or the ion exchange resins, diuretics and hemodialysis.

\section{Conclusion}

There are some case reports of life-threatening dysrhythmia and death due to rapid IV potassium infusion and oral high dose ingestion. Immediate recognition and specific treatment are vital to survive patients.

\section{Ethical issues}

Confidentiality of patient information was maintained.

\section{References}

1. Kung AW. Clinical review: thyrotoxic periodic paralysis: a diagnostic challenge. J Clin Endocrinol Metab 2006; 91(7): 2490-5. doi: 10.1210/jc.2006-0356.

2. Segal MM, Jurkat-Rott K, Levitt J, Lehmann-Horn F. Hypokalemic periodic paralysis - an owner's manual. Available from: http://simulconsult.com/resources/hypopp. html.

3. Kim JB, Kim MH, Lee SJ, Kim DJ, Lee BC. The genotype and clinical phenotype of Korean patients with familial hypokalemic periodic paralysis. J Korean Med Sci 2007; 22(6): 946-51. doi: 10.3346/jkms.2007.22.6.946.

4. Tintinalli JE, Stapczynski JS, Ma OJ, Yealy DM, Meckler GD, Cline DM. Tintinalli's Emergency Medicine. 7th ed. New York: McGraw-Hill Medical; 2011. p. 121-122.
5. Rardon DF, Fisch C. Electrolytes and the heart. In: Schlant RC, Alexander RW, eds. Hurst's the Heart. 8th ed. New York: McGraw-Hill; 1994. p. 759-74.

6. Alfonzo AV, Isles C, Geddes C, Deighan C. Potassium disorders--clinical spectrum and emergency management. Resuscitation 2006; 70(1): 10-25. doi:10.1016/j. resuscitation.2005.11.002.

7. McCann B, Hunter R, McCann J. Cocaine/heroin induced rhabdomyolysis and ventricular fibrillation. Emerg Med J 2002; 19(3): 264-5.

8. Ochoa-Gómez J, Villar-Arias A, Aresti I, Marco-Aguilar P. A case of severe hyperkalaemia and compartment syndrome due to rhabdomyolysis after drugs abuse. Resuscitation 2002; 54(1): 103-5.

9. Nathan A, Ganesh A, Godinez RI, Nicolson SC, Greeley WJ. Hyperkalemi cardiac arrest after cardiopulmonary bypass in a child with Duchenne muscular dystrophy. Anesth Analg 2005; 100(3): 672-4. doi: 10.1213/01. ANE.0000146533.21771.2F.

10. Wetherton AR, Corey TS, Buchino JJ, Burrows AM. Fatal intravenous injection of potassium in hospitalized patients. Am J Forensic Med Pathol 2003; 24(2): 128-31. doi: 10.1097/01.paf.0000068224.31539.a9.

11. Nilsson TS, Malmgren J, Knudsen K. Parallel haemodialysis and surgery saves a life after massive overdose of potassium pills. BMJ Case Rep 2012. doi: 10.1136/bcr.02.2012.5773.

12. Battefort F, Dehours E, Vallé B, Hamdaoui A, Bounes V. Suicide attempt by intravenous potassium self-poisoning: a case report. Case Rep Emerg Med 2012; 2012: 323818. doi: 10.1155/2012/323818.

13. Bertol E, Politi L, Mari F. Death by potassium chloride intravenous injection: evaluation of analytical detectability. J Forensic Sci 2012; 57(1): 273-5. doi: 10.1111/j.15564029.2011.01907.x. 